\title{
Reducing Equivalent Shuttles in Developing Porcine Myocardium: Enhanced Capacity in the Newborn Heart
}

\author{
THOMAS D. SCHOLZ, ANI STACIA L. KOPPENHAFER \\ Department of Pediatrics, Division of Pediatric Cardiology, University of Iowa. Iowa City: Iowa $522+2$
}

\begin{abstract}
The metabolic demands of the newborn heart are met primarily by glucose and lactate. Mitochondria are impermeable to the NADH produced by these cytosolic reactions. The malate/ aspartate and $\alpha$-glycerophosphate ( $\alpha$-GP) shuttles provide two pathways to transport reducing equivalents into mitochondria. The goals of this study were to compare the capacity of these shuttles in newborn and adult cardiac mitochondria and to mea-

myocardium. Newborn left ventricular myocardium had significant $\alpha$-GP shuttle activity $(44+4 \mathrm{nmol} / \mathrm{min} / \mathrm{mg}$ ) due to enhanced flavin-linked mitochondrial $\alpha$-GP dehydrogenase activity compared with adult. Interventricular differences in the $\alpha$-GP shuttle capacity were not found in newborn or adult hearts. These findings suggest a mechanism for the substrate preference of neonatal myocardium. (Pediatr Res 38: 221-227, 1995)
\end{abstract} sure the maximal activity of the mitochondrial enzymes involved in these shuttles. Shuttle and enzyme capacities were measured in isolated mitochondria from the left and right ventricular free wall of 0-3-d-old and adult pig hearts. Malate/aspartate shuttle capacity was nearly three times greater in the newborn left ventricle compared with adult (newborn, $616 \pm 24$; adult, $232+28$ $\mathrm{nmol} / \mathrm{min} / \mathrm{mg} ;$ mean $=\mathrm{SEM} ; n-8 ; p<0.00(01)$. The capacity of the malate/aspartate shuttle of the right ventricular free wall was greater than the left in the adult heart. Despite a decrease in malate/aspartate shuttle capacity, maximal activity of mitochondrial matrix enzymes involved in this pathway were increased in adult mitochondria. $\alpha$-GP shuttle activity was absent in adult

Substrate utilization by newborn myocardium is different from adult (1-3). ATP for myocardial contraction is supplied predominantly by glucose and lactate in the newborn heart, whereas adult myocardium more readily oxidizes fatty acids. To efficiently produce ATP from glucose and lactate, cytosolically derived reducing equivalents from glycolysis and lactate oxidation, such as NADH, must be transported to the mitochondria for entry into the electron transport chain. Lehninger (4) and Purvis and Lowenstein (5) have demonstrated that mitochondria are impermeable to NAIDH. Thus, other mechanisms are necessary to transport cytosolic NADH into mitochondria, particularly in the newborn heart.

Several pathways have been described that allow NADI to gain entry into the mitochondria $(6,7)$. These so-called NADH shuttles involve redox couples whose reduced pair is generated in the cytosol and diffuses or is transported into the mitochon-

Received September 15, 1994: accepted Warch 11. 1995.

Correspondence: Thomas D. Scliolz. M.D., Department of Pediatrics, Division of Pediatric Cardiology. 2852 JPP, University of Iowil. Iowa (ity. IA 52242 .

\author{
Abbreviations \\ $\alpha$-GP, $\alpha$-glycerophosphate \\ $\alpha$-GPDH, $\alpha$-glycerophosphate dehydrogenalse \\ $\alpha$-KG, $\alpha$-ketoglutarate \\ MDH, malate dehydrogenase \\ AST, aspartate aminotransferase \\ $\alpha$-KGDH, $\alpha$-ketoglutarate dehydrogenase \\ $\mathrm{m}$, mitochondrial \\ AOA, amino(oxy)acetate \\ RCR, respiratory control ratio
}

dria. Within the mitochondria, the reduced substrate is oxidized to regenerate $\mathrm{NADH}$ or other reducing equivalents that can enter the electron transport chain. In adult myocardium, the malate/aspartate shuttle (Fig. 1A) is the predominant pathway for transferring NADH into the mitochondria $(8,9)$. Oxaloacetate is reduced in the cytosol by NADH to form malate which is exchanged with $\alpha-\mathrm{KG}$ as it enters the mitochondria. Malate then reduces $\mathrm{NAD}^{-1}$ to form $\mathrm{NADH}$, and the resulting oxaloacetate is transaminated to form aspartate. Exchange of aspartate with glutamate is electrogenically driven $(10-12)$ and results in the one-way movement of NADH into the mitochondria. The cycle is completed by the cytosolic deamination of aspartate to generate oxaloacetate.

An additional pathway, the $\alpha$-GP shuttle (Fig. $1 B$ ), has been described in mammalian hepatocytes, insect flight muscle, and mammalian pancreatic $\beta$-cells $(7,13,14)$. After the reduction of dihydroxyacetone phosphate to $\alpha$-GP. $\alpha$-GP diffuses to the inner mitochondrial membrane where reduction by the membrane-bound, flavin-linked $m \alpha$-GPDH generates reduced flavin adenine dinucleotide that enters the second step of the electron 

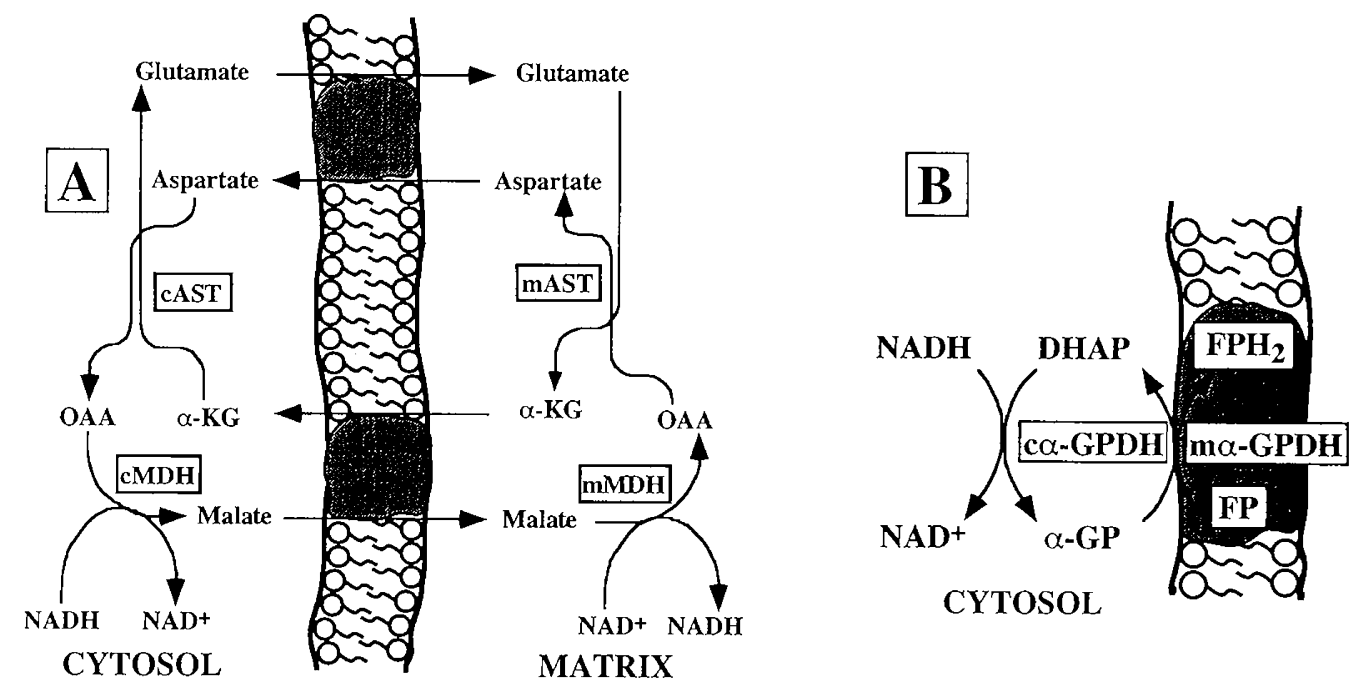

Figure 1. Reducing equivalent shuttles. Schematics of the malate/aspartate $(A)$ and $\alpha$-GP $(B)$ shuttles are shown. See text for details. Abbreviations include: $c A S T$, cytosolic aspartate aminotransferase; $O A A$, oxaloacetate; $C M D H$, cytosolic malate dehydrogenase; $D H A P$, dihydroxyacetone phosphate; $F P$, flavin phosphate; $\mathrm{FPH}_{2}$, reduced FP.

transport chain. By entering an intermediate step of the electron transport chain, the $\alpha$-GP shuttle produces one less mole of ATP per mole of transported NADH compared with the malate/aspartate shuttle.

Cytosolic derived NADH may be oxidized via a reaction with pyruvate or transported to the mitochondria for aerobic ATP production. Clearly, the glycolytically active newborn heart must efficiently shuttle $\mathrm{NADH}$ into the mitochondria to allow for maximum production of ATP. The goal of the present study was to determine whether mitochondria from newborn myocardium exhibited an increased capacity of the malate/ aspartate and $\alpha$-GP shuttles compared with adult cardiac mitochondria. Further, maximal activity of the enzymes involved in these shuttles was also investigated to identify potential steps in $\mathrm{NADH}$ transport which may be regulated during development.

\section{METHODS}

Animal preparation and isolation of mitochondria. Hearts from newborn $(0-3 \mathrm{~d}, n=8)$ and adult pigs $(n=8)$ were used for the isolated mitochondrial shuttle studies. Neonatal pigs were anesthetized with $20 \mathrm{mg} / \mathrm{kg}$ ketamine, $0.2 \mathrm{mg} / \mathrm{kg}$ acepromazine, and $2 \mathrm{mg} / \mathrm{kg}$ xylazine given s.c. Once a deep level of sedation was attained, lidocaine was generously infiltrated over the right external jugular vein which was then dissected free from surrounding tissue. The right external jugular vein was then cannulated, and a $3-\mathrm{mL}$ bolus of saturated potassium chloride was rapidly infused to arrest the heart. The heart was immediately removed through a midline sternotomy and immersed in ice-cold saline. Hearts from adult pigs were obtained from a local abattoir immediately after exsanguination and immersed in several volumes of ice-cold saline. Both the left and right ventricular free walls were dissected free of the surrounding tissue for subsequent mitochondria isolation. All animals were handled in accordance of the Animal Care and Use Guidelines of the University of Iowa.
Cardiac mitochondria were isolated using a modification of the technique described by Saks et al. (15). All isolation steps were performed at $0-4^{\circ} \mathrm{C}$. Briefly, newborn myocardium was minced with scissors and adult myocardium was passed through a meat grinder, and approximately $3 \mathrm{~g}$ of either tissue were placed in buffer containing (final concentration in $\mathrm{mM}$ ) 300 sucrose, $10 \mathrm{~N}$-2-hydroxyethylpiperazine- $N^{\prime}$-2-ethanesulfonic acid, 0.2 EDTA at $\mathrm{pH} 7.2$. Trypsin $(2.5 \mathrm{mg})$ was added to the tissue suspension and incubated at $0^{\circ} \mathrm{C}$ for $15 \mathrm{~min}$. The reaction was stopped with a 5 -fold excess of trypsin inhibitor dissolved in $20 \mathrm{~mL}$ of sucrose buffer with $1 \mathrm{mg} / \mathrm{mL}$ BSA. After decanting the fluid, the tissue was resuspended in $20 \mathrm{~mL}$ of buffer with BSA and homogenized with a Pro Scientific Pro200 homogenizer (Pro Scientific Inc., Monroe, CT) for $10 \mathrm{~s}$. The homogenate was centrifuged three times at $600 \times g$ for 10 min at $4^{\circ} \mathrm{C}$, each time saving the supernatant. The mitochondria were pelleted at $8000 \times g$ for $15 \mathrm{~min}$ and washed once in buffer with BSA. The final pellet was resuspended in the sucrose buffer without BSA.

$\mathrm{RCR}$, representing the ratio of the rate of mitochondrial oxygen consumption during state 3 activity (substrate and ADP excess) to the rate of oxygen consumption during state 4 activity (ATP present and ADP depleted), were measured in a temperature-regulated chamber with a Clark-type oxygen electrode interfaced to a Gilson OxyGraph (Gilson Medical Elec. Inc., Middleton, WI). Mitochondria were continuously stirred during these measurements. The mitochondrial suspension $(200 \mu \mathrm{L})$ was added to $1.5 \mathrm{~mL}$ of (final concentration in $\mathrm{mM}$ ) 130 potassium chloride, $20 \mathrm{~N}$-2-hydroxyethylpiperazine- $N^{\prime}-2$ ethanesulfonic acid, 2.5 magnesium chloride, 0.5 EDTA, 5 potassium phosphate, 5 glutamate, and 5 malate at pH 7.2. After stabilization of the suspension, state 3 activity was measured after addition of $580 \mu \mathrm{M}$ ADP. State 4 activity was then measured after depletion of available ADP. All mitochondria from newborn and adult left ventricular free wall 
were well coupled, demonstrating RCR greater than 10 (see "Results").

Reconstitution of the malate/aspartate shuttle. The maximal activity of the malate/aspartate shuttle was determined using the method of Cederbaum et al. (7) as modified by Dawson (16) and Sharma et al. (17). In a 3-mL plastic cuvette, $50 \mu \mathrm{L}$ of mitochondrial suspension were vigorously mixed with $2 \mathrm{~mL}$ of (final concentration in $\mathrm{mM}$ ) 300 mannitol, 10 potassium phosphate, 10 Tris, 10 potassium chloride, 5 magnesium chloride, 2 aspartate, $2 \mathrm{ADP}$, and $0.14 \mathrm{NADH}$ with 3 $\mathrm{U} / \mathrm{mL} \mathrm{MDH}$ and $2 \mathrm{U} / \mathrm{mL}$ AST at $\mathrm{pH}$ 7.4. Baseline oxidation of $\mathrm{NADH}$ was monitored at $340 \mathrm{~nm}$ at a constant temperature of $37^{\circ} \mathrm{C}$ for 4 min (Beckman DU-64 Spectrophotometer, Beckman Instruments, Inc., Fullerton, CA). Malate/aspartate shuttle activity was initiated with the addition of $4 \mathrm{mM}$ malate and 4 $\mathrm{mM}$ glutamate (final concentration), and the oxidation of $\mathrm{NADH}$ was monitored at $340 \mathrm{~nm}$ for $4 \mathrm{~min}$. The contents of the cuvette were vigorously stirred with a $3.0-\mathrm{mm}$ Teflon rod after substrate addition (more frequent stirring during data collection did not alter the measured rate of NADH oxidation). The difference between the rate of change of absorbance with and without added substrates was normalized to added mitochondrial protein to determine the shuttle capacity. For this study and others, the rate of change of absorbance was calculated using the kinetics package available on the Beckman DU-64. Curves after addition of substrate were linear over the 4-min observation period with linear regression curve fits always yielding $r$ values greater than 0.99 .

To demonstrate that the observed oxidation of NADH occurred through the malate/aspartate shuttle, all reactions were repeated with $0.4 \mathrm{mM}$ AOA added to the cuvette before baseline measurements. AOA is a competitive inhibitor of transaminases, including AST $(9,18)$, and effectively inhibits malate/aspartate shuttle activity at this concentration (9). The rate of absorbance change with and without added substrates was normalized to added mitochondrial protein to determine the shuttle activity in the presence of AOA.

Reconstitution of the $\alpha-G P$ shuttle. Studies of the maximal activity of the $\alpha$-GP shuttle were performed as described by Cederbaum et al. (7). Briefly, the buffer used for the malate/ aspartate shuttle studies was modified by excluding aspartate, $\mathrm{MDH}$, and AST and including $1 \mathrm{mM}$ ATP and $1 \mathrm{U} / \mathrm{mL}$ $\alpha$-GPDH. Baseline absorbance was followed at $340 \mathrm{~nm}$ at $37^{\circ} \mathrm{C}$ for $4 \mathrm{~min}$. The $\alpha$-GP shuttle was activated with the addition of $10 \mathrm{mM} \alpha$-GP (final concentration) and stirring of the suspension with a Teflon rod. The difference in the rate of change of absorbance with and without added $\alpha$-GP was normalized to added mitochondrial protein to determine the capacity of the $\alpha$-GP shuttle.

Tissue preparation-enzyme assays. Mitochondria were isolated from the left and right ventricular free wall from newborn $(0-3 \mathrm{~d}, n=6)$ and adult pigs $(n=6)$ as described above. The mitochondrial pellet was resuspended in $2.0 \mathrm{~mL}$ of $50 \mathrm{mM}$ potassium phosphate buffer ( $\mathrm{pH} 7.2$ ). A 1.0-mL portion of the mitochondrial suspension was incubated in $0.5 \%$ Triton $\mathrm{X}-100$ for $30 \mathrm{~min}$ at $0^{\circ} \mathrm{C}$ and then sonicated for three $1-\mathrm{min}$ intervals at $140 \mathrm{~W}$ in an ice water-filled cuphorn. The sample was then centrifuged at $48,000 \times g$ for 20 min to pellet mitochondrial membranes. The supernatant was saved for mitochondrial enzyme assays (soluble mitochondrial fraction). Another $1.0-\mathrm{mL}$ portion of the mitochondrial suspension underwent homogenization for four $10-\mathrm{s}$ intervals with the Pro Scientific Pro-200 homogenizer set on high. The sample was kept on ice during homogenization as well as during the intervals between homogenization. This sample was then centrifuged at $48,000 \times g$ for $20 \mathrm{~min}$, and the supernatant was discarded. The pellet was resuspended in $0.3 \mathrm{~mL}$ of $50 \mathrm{mM}$ potassium phosphate buffer ( $\mathrm{pH}$ 7.2) for assays of flavin-linked $\alpha-\mathrm{GPDH}$ activity (particulate mitochondrial fraction).

Specific enzyme assays. All enzyme activities were measured under saturating substrate conditions. The protein content of the soluble and particulate mitochondrial fractions was determined by the method of Lowry et al. (19).

The assay for AST was adapted from Bergmeyer (20). Briefly, the reaction catalyzed by AST between aspartate and $\alpha-\mathrm{KG}$ to form glutamate and oxaloacetate was coupled to the reaction between oxaloacetate and $\mathrm{NADH}$ catalyzed by $\mathrm{MDH}$ to form malate and $\mathrm{NAD}^{+}$. In a $1.5-\mathrm{mL}$ cuvette, $100 \mu \mathrm{L}$ of soluble mitochondrial fraction were diluted in buffer containing (final concentration in $\mathrm{mM}$ ) 90 Tris, 240 aspartate, 0.11 pyridoxal 5-phosphate, and 0.16 NADH with $1 \mathrm{U} / \mathrm{mL} \mathrm{MDH}$ and $0.5 \mathrm{U} / \mathrm{mL}$ lactate dehydrogenase. This same buffer $(880$ $\mu \mathrm{L}$ ) was added to the cuvette, and baseline absorbance at 340 $\mathrm{nm}$ and $37^{\circ} \mathrm{C}$ was monitored for $4 \mathrm{~min}$. After the baseline period, $12 \mathrm{mM} \alpha-\mathrm{KG}$ was added to the cuvette, and the absorbance at $340 \mathrm{~nm}$ was monitored for $4 \mathrm{~min}$. To determine that the cytosolic isoenzyme did not contaminate the assay for mitochondria AST in the samples, nondenaturing PAGE was performed as described previously $(20,21)$. A $2.5 \%$ polyacrylamide gel was prepared in $50 \mathrm{mM}$ Tris and $380 \mathrm{mM}$ glycine (pH 7.0). Eight microliters of sample were added to the wells, and electrophoresis was performed for $1 \mathrm{~h}$ at $15 \mathrm{~V} / \mathrm{cm}$. The gel was kept on a cooling block maintained at approximately $5^{\circ} \mathrm{C}$. After electrophoresis, the gel was warmed to $37^{\circ} \mathrm{C}$, and a solution containing (in $\mathrm{mM}$ ) 5 aspartate, $2.5 \alpha$-KG, $4 \mathrm{NAD}^{+}$, $1 \mathrm{ADP}, 1.49$ iodonitrotetrazolium chloride, and 1 glycine with $60 \mathrm{U} / \mathrm{mL}$ glutamate dehydrogenase, $0.5 \mathrm{U} / \mathrm{mL}$ diaphorase, and $1 \%$ agarose at $37^{\circ} \mathrm{C}$ was poured evenly over the gel. Once the gel solidified, it was incubated at $37^{\circ} \mathrm{C}$ for approximately $1 \mathrm{~h}$. These studies demonstrated that the soluble mitochondrial fraction was essentially devoid of any cytosolic enzyme contamination.

Total MDH activity was determined as described by Bergmeyer (22) by measuring the rate of reduction of $\mathrm{NAD}^{+}$in the presence of malate at $37^{\circ} \mathrm{C}$. Maximal enzyme activity was initiated with the addition of $25 \mathrm{mM}$ malate. $\mathrm{MDH}$ capacity was determined by the difference between the rates of change of absorbance with and without added substrate.

Flavin-linked $m \alpha$-GPDH activity was measured by following the reduction of cytochrome $c$ as described previously (23). The assay buffer consisted of (in $\mathrm{mM}$ ) 100 potassium phosphate, 5 EDTA, 1 potassium cyanide, 0.1 phenazine methosulfate, and 0.09 cytochrome c. $100 \mu \mathrm{L}$ of particulate mitochondrial fraction were added to $2.3 \mathrm{~mL}$ of the buffer in a $3-\mathrm{mL}$ plastic cuvette. At $37^{\circ} \mathrm{C}$, the baseline absorbance at $550 \mathrm{~nm}$ was followed for $4 \mathrm{~min}$. $\alpha$-GP was then added $(25 \mathrm{mM}$ final 
concentration), and the absorbance at $550 \mathrm{~nm}$ was measured for an additional $4 \mathrm{~min}$. The difference in the rate of change of absorbance before and after adding $\alpha$-GP was divided by the mitochondrial protein added to the cuvette to determine the capacity of $m \alpha-G P D H$.

It has been shown that $\alpha-\mathrm{KGDH}$ may regulate flux through the malate/aspartate shuttle (8). Thus, $\alpha-\mathrm{KGDH}$ activity was measured on each of the soluble mitochondrial fractions using the technique described by Jackson and Singer (24). This method follows the reduction of $\mathrm{NAD}^{+}$with $\alpha-\mathrm{KG}$ and $\mathrm{CoA}$ to form succinyl CoA and NADH. The buffer solution contained (in $\mathrm{mM}$ ) 50 3-[ $N$-morpholino]propanesulfonic acid, 2 magnesium chloride, 0.5 calcium chloride, 2.6 cysteine, 0.2 thiamine pyrophosphate, $0.12 \mathrm{CoA}, 2 \mathrm{NAD}^{+}$at $\mathrm{pH} 7.6$. The rate of change of absorbance at $340 \mathrm{~nm}$ at $37^{\circ} \mathrm{C}$ after addition of $\alpha-\mathrm{KG}$ (2 $\mathrm{mM}$ ) was followed for $4 \mathrm{~min}$ and subtracted from the baseline rate to determine maximal $\alpha-\mathrm{KGDH}$ activity.

Statistics. All data are expressed as mean \pm SEM. Comparison of NADH shuttle capacities and specific enzyme activities between newborn and adult samples was made using an unpaired, two-tailed $t$ test. Differences between left and right ventricular values were assessed using a paired, two-tailed $t$ test. A Bonferroni correction was applied to all determined $p$ values to correct for the duplicate comparisons made on the same data sets.

\section{RESULTS}

Isolated mitochondria NADH shuttle studies. Mitochondria from the left and right ventricular free wall of both newborn and adult porcine hearts remained well coupled after isolation. As has been found by previous investigators $(25,26)$, the RCR of mitochondria from newborn myocardium was significantly greater than adult (Table 1). The RCR was similar for right and left ventricular newborn samples, whereas the RCR of mitochondria from adult right ventricular free wall was significantly greater than left ventricular samples (Table 1 ).

The cytosolic components of the malate/aspartate and $\alpha$-GP shuttles were provided at saturating concentrations to isolated cardiac mitochondria to determine the capacity of these NADH-transporting mechanisms (see "Methods"). Mitochondria isolated from the newborn left ventricular free wall myocardium demonstrated nearly three times the capacity to oxidize NADH through the malate/aspartate shuttle compared with mitochondria isolated from adult myocardium (Fig. $2 A$ ). After addition of $0.4 \mathrm{mM} \mathrm{AOA}$, a potent transaminase inhibitor, both groups of mitochondria showed significant levels of inhibition of the malate/aspartate shuttle (Fig. $2 B$ ). In the

Table 1. Mitochondrial RCR (oxygen consumption during state 3 divided by state 4 oxygen consumption) determined using glutamate and malate as substrates

\begin{tabular}{lll}
\hline Age & \multicolumn{1}{c}{ LV } & \multicolumn{1}{c}{ RV } \\
\hline Newborn & $25.7 \pm 2.4$ & $28.0 \pm 2.0$ \\
Adult & $14.4 \pm 1.0^{*}$ & $19.7 \pm 1.7 \dagger$
\end{tabular}

Mitochondria wcre isolated from the left and right ventricular free walls of newborn and adult porcinc hearts. All data are mean $\pm \mathrm{SEM} ; n=8$.

$* p<0.05$ versus newborn left ventricular (LV) mitochondria.

$\dagger p<0.05$ versus newborn right ventricular (RV) and adult $\mathrm{LV}$ mitochondria.
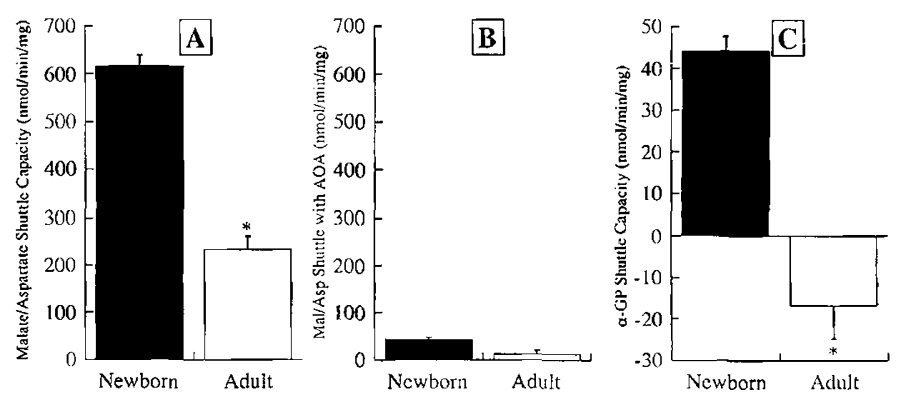

Figure 2. Reducing equivalent shuttle capacities in isolated milochondria. Isolated mitochondria from the newborn or adult porcine left ventricular free wall were incubated in the presence of saturating concentrations of the cytosolic components of the malate/aspartate $(A$ and $B)$ or $\alpha$-GP shuttle $(C)$, and the rate of NADH oxidation was measured (sce "Methods"). $A$, The malate/aspartate shuttle capacity was significantly greater in mitochondria isolated from newborn myocardium compared with adult $(p<0.0001 ; n=8)$. $B$, adding the transaminase inhibitor, AOA, to assay conditions identical to $A$, resulted in limited NADH oxidation suggesting that the majority of NADH oxidation in $A$ was occurring through the malatc/aspartate shuttlc. $C, \alpha$-GP shuttle capacity was significantly greater in newborn cardiac mitochondria compared with adult $(p<0.0001 ; n=8)$. Adult $\alpha$-GP shuttle was no different from zero (by paired $t$ test, $p>0.05$ ).

presence of added $\mathrm{AOA}$, the percent of the uninhibited rate of NADH oxidation was 7.1 and $6.3 \%$ in the newborn and adult mitochondria, respectively.

Malate/aspartate shuttle capacity was significantly greater in mitochondria isolated from newborn right ventricle compared with newborn left ventricular samples (Table 2). Although adult right ventricular mitochondria followed this trend, the interventricular difference did not reach significance.

An age-related decrease in NADH oxidation was also found when newborn and adult cardiac mitochondria were incubated in the presence of the cytosolic components of the $\alpha$-GP shuttle (Fig. 2C). The $\alpha$-GP shuttle activity of adult mitochondria was no different from baseline based on a paired $t$ test comparing baseline and postsubstrate rates of change of NADH oxidation ( $p>0.05$ ). Surprisingly, the $\alpha$-GP shuttle was active in newborn cardiac mitochondria and significantly greater than the adult. No interventricular difference was found in $\alpha$-GP shuttle capacity in either newborn or adult samples (Table 2).

NADH shuttle enzyme activity studies. Assays for the maximal activity of the individual enzymes involved in the malate/ aspartate and $\alpha$-GP shuttles were performed to identify age specific changes in enzyme activities that could explain the developmental differences in NADH shuttle capacities.

Table 2. Malate/aspartate (Mal/Asp) and $\alpha$-GP shuttle capacities of mitochondria isolated from the right and left ventricular free walls of newborn and adult porcine heart

\begin{tabular}{cccccr}
\hline & \multicolumn{2}{c}{ Newborn } & & \multicolumn{2}{c}{ Adult } \\
\cline { 2 - 3 } \cline { 5 - 6 } Mitochondria & Mal/Asp & $\alpha$-GP & & Mal/Asp & $\alpha$-GP \\
\hline LV & $616 \pm 24 *$ & $44 \pm 4 \dagger$ & & $232 \pm 28$ & $-17 \pm 8$ \\
RV & $676 \pm 22 \ddagger$ & $49 \pm 3 \ddagger$ & & $287 \pm 30$ & $2 \pm 5$ \\
\hline
\end{tabular}

All data are mean $\pm \mathrm{SEM}$ in $\mathrm{nmol} / \mathrm{min} / \mathrm{mg}$ mitochondrial protein; $n=8$.

$* p<0.05$ vs newborn right ventricular (RV) and adult left ventricular (LV) mitochondria.

$t p<0.05$ vs adult LV milochondria.

$\ddagger p<0.05$ vs adult $\mathrm{RV}$ mitochondria. 
Maximal activity of the mitochondrial isoenzymes of the malate/aspartate shuttle tended to be greater in the adult. The maximal activity of the mitochondrial isoform of AST (mAST) and $\mathrm{mMDH}$ were significantly greater in mitochondria isolated from adult left ventricular myocardium compared with the newborn left ventricle (Table 3). In the newborn heart, right ventricular mAST capacity was greater than newborn left ventricular mAST activity. The activity of the potential malate/ aspartate shuttle regulatory enzyme $\alpha-\mathrm{KGDH}$ (8) was also. greater in adult left ventricular mitochondria compared with newborn (Table 3). No difference in $\alpha$-KGDH maximal activity was found between newborn and adult right ventricular mitochondria due to an increase in the maximal activity of newborn right ventricular samples. The observation that malate/aspartate shuttle capacity is greater in isolated newborn mitochondria (Fig. $2 A$ ), despite the decreased maximal activity of the newborn mitochondrial malate/aspartate shuttle enzymes (Table 3), suggests that the maximal activity of the membrane malate $/ \alpha-\mathrm{KG}$ and aspartate/glutamate translocases may be increased in newborn cardiac mitochondria.

The maximal activity of the flavin-linked $m \alpha-\mathrm{GPDH}$ was significantly greater in newborn right and left ventricular mitochondria than adult (Fig. 3). Thus, the maximal rate at which newborn mitochondria can oxidize $\alpha$-GP and bring protons into the electron transport chain is significantly greater than the adult.

\section{DISCUSSION}

The present study demonstrated a significant increase in the capacity of the malate/aspartate and $\alpha$-GP shuttles present in isolated mitochondria from newborn myocardium compared with adult tissue. Malate/aspartate shuttle capacity was nearly three times greater in neonatal mitochondria. Maximal $\alpha$-GP shuttle activity was also significantly greater in mitochondria isolated from newborn myocardium. Adult left ventricular mitochondria demonstrated negligible $\alpha$-GP shuttle activity. In newborn mitochondria, $\alpha$-GP shuttle capacity was approximately $7 \%$ of newborn malate/aspartate shuttle levels. Thus, although the $\alpha$-GP shuttle is active in newborn myocardium, the significance of this pathway relative to the malate/aspartate shuttle appears to be limited.

Few studies have been performed to examine the capacity of the NADH shuttles during development. Studying young rat tissues, Sharma et al. (17) found increased malate/aspartate

Table 3. Maximal activity of the mitochondrial enzymes involved in the malate/aspartate shuttle were determined under saturating substrate conditions (see "Methods")

\begin{tabular}{llllll}
\hline & \multicolumn{2}{c}{ Newborn } & & \multicolumn{2}{c}{ Adult } \\
\cline { 2 - 3 } \cline { 5 - 6 } Enzyme & \multicolumn{1}{c}{ LV } & \multicolumn{1}{c}{$\mathrm{RV}$} & & $\mathrm{LV}$ & \multicolumn{1}{c}{$\mathrm{RV}$} \\
\hline mAST & $41.0 \pm 3.4^{*}$ & $52.8 \pm 5.2 \dagger$ & & $63.3 \pm 4.4$ & $62.0 \pm 5.2$ \\
$\mathrm{mMDH}$ & $45.2 \pm 4.5^{*}$ & $48.0 \pm 6.3$ & & $63.5 \pm 5.0$ & $63.4 \pm 5.5$ \\
$\alpha$-KGDH & $2.08 \pm 0.10^{*}$ & $2.90 \pm 0.21 \dagger$ & & $3.10 \pm 0.42$ & $3.30 \pm 0.43$ \\
\hline
\end{tabular}

Mitochondria were isolated from left and right ventricular myocardium of newborn and adult porcine hearts. All data are mean \pm SEM in $\mu \mathrm{mol} / \mathrm{min} / \mathrm{mg}$ mitochondrial protein; $n=6$.

$* p<0.05$ vs adult left ventricular (LV) mitochondrial enzyme capacity.

$\dagger p<0.05$ vs newborn LV mitochondrial enzyme capacity.

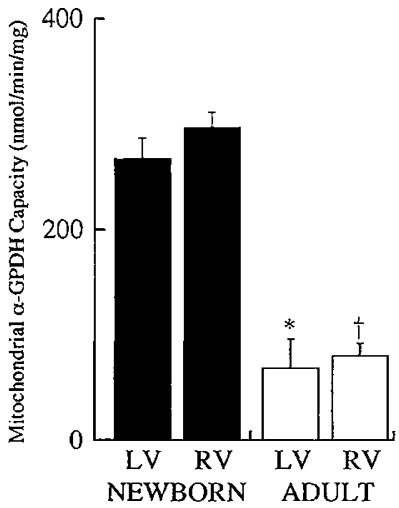

Figure 3. $\alpha$-GP shuttle enzyme capacities. The maximal activity of the mitochondrial enzyme involved in the $\alpha$-GP shuttle ( $\alpha$-GPDH) was measured under substrate saturating conditions. The flavin-linked mitochondrial $\alpha$-GPDH capacity was significantly greater in newborn myocardium compared with adult $\left({ }^{*} p<0.001\right.$ vs newborn LV; $\dagger p<0.001$ vs newborn RV; $n=6$ ). Data are normalized to milligrams of mitochondrial protein. $L V$, left ventricle; $R V$, right ventricle.

shuttle activity in 15-d-old kidney compared with 30-d-old, whereas the opposite relationship was found in liver. Schantz and Kallman (27) and Schantz and Henriksson (28) examined the capacity of both the malate/aspartate and $\alpha$-GP shuttles in human skeletal muscle. Neither strength nor endurance training altered the maximal activity of the NADH shuttles. Although much of the initial work on the malate/aspartate shuttle, and to a lesser extent the $\alpha$-GP shuttle, was performed using cardiac mitochondria $(8,10,29)$, to our knowledge, the present study is the first to examine the developmental changes of the NADH shuttles in myocardium.

Mechanisms of increased NADH shuttle capacities. Assays of the maximal activity of the mitochondrial enzymes involved in the malate/aspartate and $\alpha$-GP shuttles provided insights into the mechanisms by which the capacity of these pathways decreased during development. The capacities of the mitochondrial matrix isoenzymes of AST and MDH (mAST and $\mathrm{mMDH}$ ) that form the mitochondrial portion of the malate/ aspartate shuttle were found to be significantly increased in adult mitochondria, suggesting that the intramitochondrial oxidation of malate and the deamination of glutamate may not be limiting in the adult relative to the newborn. It has been proposed that, by limiting the availability of $\alpha$-KG for exchange with cytosolic malate (see Fig. 1), flux through $\alpha$-KGDH could provide a level of control over the flux through the malate/aspartate shuttle $(8,30)$. The present study demonstrated an increase in the maximal activity of $\alpha-\mathrm{KGDH}$ in adult mitochondria which suggests that increased flux through this step in the citric acid cycle could limit availability of $\alpha-\mathrm{KG}$ and decrease the maximal activity of the malate/aspartate shuttle in adult myocardium by this mechanism.

In the present study, reduced shuttle capacity was found in intact, isolated adult mitochondria (Fig. 2) despite an increase in $\mathrm{mAST}$ and $\mathrm{mMDH}$ maximal activities (Table 3). The decrease in malate/aspartate shuttle capacity found with adult mitochondria in the presence of saturating concentrations of the cytosolic components suggests that the capacity of the malate/ $\alpha-K G$ and/or glutamate/aspartate translocases is de- 
creased in adult mitochondria compared with those of the newborn. The electrogenically driven aspartate/glutamate translocase is the site that drives the malate/aspartate shuttle in the direction of intramitochondrial NADH movement $(10,12$, 31,32 ). Therefore, both the capacity of the translocase protein and the malate/aspartate shuttle are sensitive to the mitochondrial membrane potential. Although the mitochondrial membrane potential is thought not to vary with age (33), the role of the translocases in the enhanced shuttle capacity in newborn mitochondria is currently not known.

The role of the inner mitochondrial membrane protein that is part of the $\alpha$-GP shuttle was assessed in the current study. The flavin-linked $m \alpha$-GPDH appeared to be the rate-limiting step of the $\alpha$-GP shuttle in adult cardiac mitochondria. The activity of this enzyme in adult mitochondria was only $25 \%$ of that found in newborn tissue (Fig. 3).

The present study has identified or suggested several potential enzymes in the malate/aspartate and $\alpha$-GP shuttle pathways whose capacities may be altered during development to enhance movement of $\mathrm{NADH}$ into mitochondria in newborn myocardium. The specific modifications to the proteins involved, whether a decrease in total protein or inhibition of activity, have not been identified from these studies. It is known that the activity of many dehydrogenases can be increased by calcium (34). Calcium up-regulation of flavinlinked $\mathrm{m} \alpha$-GPDH has been suggested to increase $\alpha$-GP shuttle activity in glucose-stimulated pancreatic $\beta$-cells (14), whereas calcium-enhanced aspartate efflux from liver cells isolated from rats after acute ethanol exposure has been implicated in increasing malate/aspartate shuttle capacity (35). Recent studies that have demonstrated that newborn cardiac mitochondria have nearly twice the maximal rate calcium uptake (33) suggest that altered calcium handling in newborn myocardium could explain the increase in shuttle capacities in neonatal mitochondria.

Myocardial substrate utilization and NADH shuttles. It is generally accepted that the newborn heart relies on glucose and lactate as its main source of metabolic fuel $(1,36)$. Elegant studies by Lopaschuk et al. (37) found that, when 1-d-old rabbit hearts were perfused with glucose, lactate, and palmitate as substrates, nearly half of the ATP produced was derived from glucose entering glycolysis with an additional $25 \%$ coming from lactate oxidation. Similar results have been found in newborn porcine myocardium $(38,39)$. Using isolated, perfused neonatal porcine heart, it has been shown that less than a third of myocardial ATP is produced via fatty acid oxidation when glucose and lactate are also available (38). Even at increased workloads, the proportion of ATP produced via fatty acid oxidation remained stable (38). The mechanism of substrate preference has not been determined, although it may relate to increased levels of lactate and decreased levels of fatty acids circulating in the fetus $(40,41)$ or a reduction in carnitine acyltransferase I activity in newborn heart (42). This enzyme is needed to allow mitochondria to use long-chain fatty acids. The present study demonstrated increased capacity of both the malate/aspartate and $\alpha$-GP shuttles in newborn myocardium, two systems by which cytosolically derived NADH can be transported into the mitochondria. Although enhanced NADH shuttle capacity in isolated mitochondria may not determine the preference of intact newborn myocardium for glucose and lactate, the efficient removal of reducing equivalents may contribute to the enhanced flux through the metabolic pathways of glucose and lactate.

Study limitations. Developmental changes in several of the enzymes measured in the current study have been demonstrated by others. Similar to the results of the present study, maximal cardiac mAST activity has also been shown to increase with age $(43,44)$. Although not tested in these previous studies, contamination of the mitochondrial fraction by cytosolic isoenzymes was examined in the present study using gel electrophoresis to separate cytosolic and mitochondrial AST isoenzymes. These studies demonstrated that minimal contamination of the mitochondrial fraction by the cytosolic enzymes occurred.

Only two time points were examined in the current study. A complex pattern of changes in $\mathrm{NADH}$ shuttle and shuttle enzyme activities have been found in other tissues and species $(17,45)$. Further studies at various ages, including prenatal time points, of both the malate/aspartate and $\alpha$-GP shuttle activities may provide further insights into potential mechanisms (e.g. diet, oxygenation) that may regulate the development of these pathways.

Porcine myocardium was used in the present study because the newborn heart is of sufficient size to allow isolation of mitochondria from the left and right ventricular free walls and the enzyme assays described above. Compared with other species, the newborn porcine heart has been shown to have increased ability to metabolize fatty acids $(1,39)$. Using isolated perfused newborn porcine hearts, Ascuitto et al. (38) and Werner et al. (39) have shown that the $0-3$-d-old porcine heart can readily use fatty acids. However, the percentage of myocardial oxygen consumption (or ATP production) accounted for by fatty acid oxidation was $28-29 \%$ with the majority of oxygen consumption provided by glucose and lactate in the perfusate (38). Studies of the NADH shuttle capacities of neonatal hearts from other mammals which rely more heavily on glucose or lactate for ATP production may provide further insights into the relationship between $\mathrm{NADH}$ shuttle capacities and myocardial substrate utilization.

\section{CONCLUSIONS}

In its dependence on glucose and lactate for energy production, the newborn heart must have a mechanism to remove $\mathrm{NADH}$ from the cytosolic compartment. Because the inner mitochondrial membrane is impermeable to $\mathrm{NADH}(4,5)$, various NADH shuttles have been described that provide pathways for reduced intermediates to enter the mitochondria for reoxidation (6). The present study found that the capacity of two of these NADH shuttles, the malate/aspartate and $\alpha$-GP shuttles, are significantly increased in newborn right and left ventricular myocardium compared with adult. Further, the results suggested that the inner mitochondrial membrane translocase proteins of the malate/aspartate shuttle are increased in the newborn heart. Increased capacity of the $\alpha$-GP shuttle in neonatal myocardium appeared to be due to increased flavin- 
linked $m \alpha-G P D H$ activity. These findings add to the understanding of developmental changes in myocardial metabolism and specifically suggest a mechanism for the substrate preference of newborn myocardium.

Acknowledgments. The authors thank Dr. William Rhead for his intellectual support and generous loans of essential equipment and Drs. Brian Schutte and David Fisher for their critical review of the manuscript.

\section{REFERENCES}

1. Lopaschuk GD, Collins-Nakai RL, Itoi T 1992 Developmental changes in energy substrate use by the heart. Cardiovasc Res 26:1172-1180

2. Girard J, Ferre P, Pegorier J-P, Duee P-H 1992 Adaptations of glucose and fatty acid metabolism during perinatal period and suckling-weanling transition. Physiol Rev 72:507-562

3. Jones CT, Rolph TP 1985 Metabolism during fetal life: a functional assessment of metabolic development. Physiol Rev 65:357-430

4. Lehninger AL 1951 Phosphorylation coupled to oxidation of dihydrodiphosphopyridine nucleotide. J Biol Chem 190:345-359

5. Purvis JL, Lowenstein JM 1961 The relationship between intra- and extramitochondrial pyridine nucleotides. J Biol Chem 236:2794-2803

6. Dawson AG 1979 Oxidation of cytosolic NADH formed during aerobic metabolism in mammalian cells. Trends Biochem Sci 4:171-176

7. Cederbaum Al, Licber CS, Beattie DS, Rubin E 1973 Characterization of shuttle mechanisms for the transport of reducing equivalents into mitochondria. Arch Biochem Biophys 158:763-781

8. LaNoue KF, Williamson JR 1971 Interrelationships between malate-aspartate shuttle and citric acid cycle in rat heart mitochondria. Metab Clin Exp 20:119-140

9. Bunger R, Glanert S, Gerlach E 1980 Inhibition by (aminooxy)acetate of the malate-aspartate cycle in the isolated working guinea pig heart. Z Physiol Chem 361:907-914

10. LaNoue KF, Meijer AJ, Brouwer A 1974 Evidence for electrogenic aspartate transport in rat liver mitochondria. Arch Biochem Biophys 161:544-550

11. LaNoue KF, Bryla J, Bassett DJ 1974 Energy-driven aspartate efflux from heart and liver mitochondria. J Biol Chem 249:7514-7521

12. LaNoue KF, Tischler ME 1974 Electrogenic characteristics of the mitochondrial glutamate-aspartate antiporter. J Biol Chem 249:7522-7528

13. Estabrook RW, Sacktor B 1958 [apha]-Glycerophosphate oxidase of flight muscle mitochondria. J Biol Chem 233:1014-1019

14. Meglasson MD, Smith KM, Nelson D, Erecinska M $1989 \alpha$-Glycerophosphate shuttle in a clonal $\beta$-cell line. Am J Physiol 256:E173-E178

15. Saks VA, Kuznetsov AV, Kupriyanov VV, Miceli MV, Jacobus WE 1985 Creatine kinase of rat heart mitochondria. J Biol Chem 260:7757-7764

16. Dawson AG 1982 Rapid oxidation of $\mathrm{NADH}$ via the reconstituted malate-aspartate shuttle in systems containing mitochondrial and soluble fractions of rat liver: Implications for ethanol metabolism. Biochem Pharmacol 31:2733-2738

17. Sharma R, Dey S, Verma R 1992 Age-specific development of malate-aspartate shuttle in the liver and kidney of mice. Biochem Int 27:1059-1066

18. Hopper S, Segal HL 1962 Kinetic studies of rat liver glutamic-alanine transaminase. J Biol Chem 237:3189-3195

19. Lowry OH, Rosebrough NJ, Farr AL, Randall RJ 1951 Protein measurement with the folin phenol reagent. J Biol Chem 193:265-275

20. Bergmeyer HU 1983 Methods of Enzymatic Analysis, Vol. III. Enzymes 1: Oxidoreductases, Transferases, 3rd Ed. Verlag Chemie, Weinheim, pp 416-444

21. Schantz PG, Sjoberg B, Svedenhag J 1986 Malate-aspartate and $\alpha$-glycerophosphate shuttle enzyme levels in human skeletal muscle: Methodological considerations and effect of endurance training. Acta Physiol Scand 128:397-407
22. Bergmeyer HU 1983 Methods of Enzymatic Analysis, Vol. III. Enzymes 1: Oxidoreductases, Transferases, 3rd Ed. Verlag Chemie, Weinheim, pp 163-175

23. Kubista V, Kubistova J, Pette D 1971 Thyroid hormone induced changes in the enzyme activity pattern of energy-supplying metabolism of fast (white), slow (red), and heart muscle of the rat. Eur J Biochem 18:553--560

24. Jackson RH, Singer TP 1983 Inactivation of the 2-ketoglutarate and pyruvate dehydrogenase complexes of beef heart by branched chain keto acids. J Biol Chem 258:1857-1865

25. Wells RJ, Friedman WF, Sobel BE 1972 Increased oxidative metabolism in the fetal and newborn lamb heart. Am J Physiol 222:1488-1493

26. Goodwin CW, Mela L, Deutsch C, Forster RE, Miller LD, Delivoria-Papadopoulos M 1976 Development and adaptation of heart mitochondrial respiratory chain function in fetus and in newborn. Adv Exp Med Biol 75:713-719

27. Schantz PG, Kallman M 1989 NADH shuttle enzymes and cytochrome $b_{5}$ reductase in human skeletal muscle: Effect of strength training. J Appl Physiol 67:123-127

28. Schantz PG, Henriksson J 1987 Enzyme levels of the NADH shuttle systems: Measurements in isolated muscle fibres from humans of differing physical activity. Acta Physiol Scand 129:505-515

29. Williamson JR, Safer B, LaNoue KF, Smith CM, Walajtys E 1973 Mitochondrialcytosolic interactions in cardiac tissue: Role of the malate-aspartate cycle in the removal of glycolytic NADH from the cytosol. Symp Soc Exp Biol 27:241281

30. LaNoue KF, Walajtys EI, Williamson JR 1973 Regulation of glutamate metabolism and interactions with the citric acid cycle in rat heart mitochondria. J Biol Chem 248:7171-7183

31. Tischler ME, Pachence J, Williamson JR, LaNoue KF 1976 Mechanism of glutamateaspartate translocation across the mitochondrial inner membrane. Arch Biochem Biophys 173:448-462

32. Kunz WS, Davis EJ 1991 Control of reversible intracellular transfer of reducing potential. Arch Biochem Biophys 284:40-46

33. Wolf WJ, Rex KA, Geshi E, Sordahl LA 1991 Postnatal changes in heart mitochondrial calcium and energy metabolism. Am J Physiol 261:H1-H8

34. Denton RM, McCormack JG $1990 \mathrm{Ca}^{2+}$ as a second messenger within mitochondrial of the heart and other tissue. Annu Rev Physiol 52:451-466

35. Sugano T, Handier JA, Yoshihara H, Kizaki Z, Thurman RG 1990 Acute and chronic ethanol treatment in vivo increases malate-aspartate shuttle capacity in perfused rat liver. J Biol Chem 265:21549-21553

36. Fisher DJ 1984 Oxygenation and metabolism in the developing heart. Scmin Perinatol $8: 217-225$

37. Lopaschuk GD, Spafford MA, Marsh DR 1991 Glycolysis is the predominant source of myocardial ATP production immediately after birth. Am J Physiol 261:H1698H1705

38. Ascuitto RJ, Ross-Ascuitto NT, Chen V, Downing SE 1989 Ventricular function and fatty acid metabolism in neonatal piglet. Am J Physiol 256:H9-H15

39. Werner JC, Sicard RE, Schuler HG 1989 Palmitate oxidation by isolated working fetal newborn pig hearts. Am J Physiol 256:E315-E321

40. Warshaw JB 1979 Fatty acid metabolism during development. Semin Perinatol 3:131-139

41. Comline RS, Silver M 1976 Some aspects of fetal and uteroplacental metabolism in cows with indwelling umbilical and uterine vascular catheters. J Physiol 260:571-586

42. Warshaw JB 1972 Cellular energy metabolism during fetal development. IV. Fatty acid activation, acyl transfer and fatty acid oxidation during development of the chick and rat. Dev Biol 28:537-544

43. Rolph TP, Jones CT, Parry D 1982 Ultrastructural and enzymatic development of fetal guinea pig heart. Am J Physiol 243:H87-H93

44. Herzfeld A, Greengard O 1971 Aspartate aminotransferase in rat tissues: Changes with growth and hormones. Biochim Biophys Acta 237:88-98

45. Edwards EM, Dhand UK, Jeacock MK, Shepherd DAL 1975 Activities of enzymes concerned with pyruvate and oxaloacetate metabolism in the heart and liver of developing sheep. Biochim Biophys Acta 399:217--227 